\title{
Recent results from Higgs boson physics at the LHC
}

\author{
Florencia Canelli*t \\ University of Zurich \\ E-mail: canelliephysik.uzh.ch
}

\begin{abstract}
Results on Higgs boson physics with proton-proton collision data at $\sqrt{s}=13 \mathrm{TeV}$ collected by the ATLAS and CMS detectors at the Large Hadron Collider corresponding to an integrated luminosity up to $15 \mathrm{fb}^{-1}$ taken during 2015 and part of 2016 are presented. Standard Model Higgs boson measurements in different production and decay channels such as $H \rightarrow \gamma \gamma, Z Z, W W, \mu \mu$, $b \bar{b}$, as well as Beyond-Standard-Model searches for first indications of an extended Higgs boson sector, are summarized. Measurements of the fiducial and differential cross sections for Higgs boson production in the $H \rightarrow \gamma \gamma$ and $H \rightarrow Z Z^{*} \rightarrow 4 l$ channels are also included.
\end{abstract}

38th International Conference on High Energy Physics

3-10 August 2016

Chicago, USA

* Speaker.

${ }^{\dagger}$ A footnote may follow. 


\section{Introduction}

The discovery of a Higgs boson at a mass of about $125 \mathrm{GeV}$ by the ATLAS and CMS experiments [1,2] at the Large Hadron Collider (LHC) has been a milestone for elementary particle physics. Since this fundamental discovery, the ATLAS and CMS collaboration have scrutinized the complete LHC Run I dataset at $\sqrt{s}=7$ and $\sqrt{s}=8 \mathrm{TeV}$ of pp collisions (about $25 \mathrm{fb}^{-1}$ ), and focused on determining the properties of the new state and searching for potential additional particles in the electroweak symmetry breaking sector. In order to determine its properties, and to establish whether they are in precise agreement with the expectations of the Standard Model (SM), detailed measurements of the coupling strengths and structure, spin/CP, mass, and decay widths have been made $[3,4]$. Current measurements of properties are so far consistent with a SM Higgs-boson. However, a wide range of Beyond-Standard-Model (BSM) scenarios are still allowed which can be potentially revealed by direct searches for additional Higgs bosons.

These proceedings describe the first results on Higgs boson physics SM measurements and BSM searches based on Run 2 data delivered by the LHC and collected by ATLAS and CMS at $\sqrt{s}$ $=13 \mathrm{TeV}$ from $2015\left(\sim 3 \mathrm{fb}^{-1}\right)$ to mid $2016\left(\sim 13 \mathrm{fb}^{-1}\right)$.

\section{SM Higgs boson}

The main Higgs boson production modes consist of gluon-gluon fusion $(\mathrm{ggH})$, vector-boson fusion $(V B F)$, associated vector bosons $(V H)$ and associated top quark pairs $(t \bar{t} H)$ and singletop quarks $(t H)$, as according to their SM cross sections in decreasing rate. The experimental analyses are categorized and optimized according to different Higgs boson decays given that their SM backgrounds are widely different. All production and decay modes have been studied during Run 1.

ATLAS and CMS experiments combined their Higgs-boson mass measurements in fully reconstructed $H \rightarrow Z Z \rightarrow 4 l$ and $H \rightarrow \gamma \gamma$ channels to produce the Run 1 LHC average mass value of $m_{H}=125.09 \pm 0.24 \mathrm{GeV}$ [4]. Measurements of the couplings to SM particles such as $W$ and $Z$ bosons, $t, b$ and $\tau$ fermions, as well as $\mu$ leptons from both experiments were also combined. The combination of the measurement of the couplings was based on the measured $\sigma \times \operatorname{BR}(H \rightarrow X X)$ for all investigated Higgs boson decay modes, i.e. $H \rightarrow Z Z, H \rightarrow \gamma \gamma, H \rightarrow W W, H \rightarrow b \bar{b}, H \rightarrow \tau \tau$ and $H \rightarrow \mu \mu$ [3]. The combined signal yield relative to the SM expectation is $\mu=1.09 \pm 0.11$, remaining compatible with the SM expectation within its uncertainty. The combination of the two experiments leads to observed significances of the $V B F$ production process and of the $H \rightarrow \tau \tau$ decay at the level of $5.4 \sigma$ and $5.5 \sigma$, respectively. The combined significance for the $t \bar{t} H$ process was expected to be $2.0 \sigma$, whereas an excess of $4.4 \sigma$ was measured. Precise measurements of the Higgs boson coupling strengths to $\mathrm{SM}$ vector bosons and fermions constitute a stringent probe of various BSM Higgs scenarios. Measurements of Higgs boson couplings in Run 1 so far remain consistent with their SM predictions and are found to follow the expected mass scaling. Multiple $J^{C P}$ scenarios of the observed Higgs boson were probed during Run 1 and results exclude all considered non-SM spin hypotheses at more than $99 \%$ confidence level (CL) [5, 6]. 


\section{1 $H \rightarrow \gamma \gamma$}

Despite the small branching ratio predicted by the SM $(\sim 0.23 \%)$, the $H \rightarrow \gamma \gamma$ channel provides a clean final state with small backgrounds, which mainly consist of irreducible $\gamma \gamma, \gamma+$ jet, and dijet events, making it one of the most sensitive channels. The dominant uncertainty in this channel is the photon energy scale and resolution as well as the background-choice bias in the fit. This analysis is optimized in different production modes, $g g F, V B F, t \bar{t} H, V H$. Figure 1 shows the $m_{\gamma \gamma}$ distributions from ATLAS and CMS experiments used to extract the signal contribution from $13.3 \mathrm{fb}^{-1}$ and $12.9 \mathrm{fb}^{-1}$ of pp collision data, respectively [7, 8]. Signal strengths and fiducial cross sections calculated with event yields corrected for detector inefficiency and resolution for minimal theoretical modeling are shown in Table 1. Differential cross section measurements were done by ATLAS and some of these studies are shown in Fig. 2.
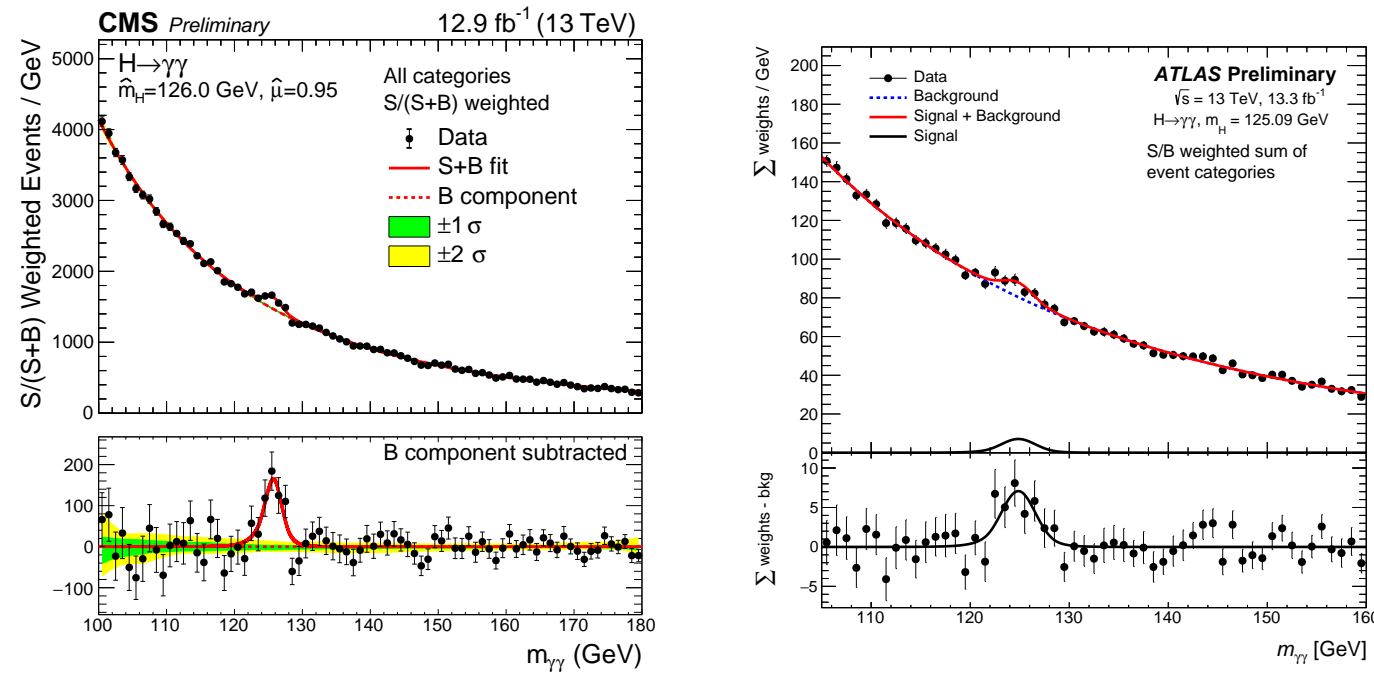

Figure 1: CMS (left) and ATLAS (right) invariant mass spectrum combining all production mode categories. Data points (black) and signal plus background model fits for all event categories summed, weighted by their sensitivity. The bottom inset displays the residuals of the data with respect to the fitted background component [7, 8].

Table 1: Fiducial cross section measurements $H \rightarrow \gamma \gamma[7,8]$

$\begin{array}{llll}\sqrt{s}=13 \mathrm{TeV} & \text { Measurement }(\mathrm{fb}) & \text { SM prediction }(\mathrm{fb}) & \mu \text { signal strength } \\ \text { ATLAS }\left(13.3 \mathrm{fb}^{-1}\right) & 43.2 \pm 14.9 \text { (stat) } \pm 4.9(\text { syst }) & 62.8_{-4.4}^{+3.4}(\mathrm{~N} 3 \mathrm{LO}+\mathrm{XH}) & 0.85_{-0.20}^{+0.22} \\ \text { CMS }\left(12.9 \mathrm{fb}^{-1}\right) & 69_{-22}^{+16}(\text { stat })_{-6}^{+8} \text { (syst) } & 73.8 \pm 3.8 & 0.95_{-0.18}^{+0.21}\end{array}$

$2.2 H \rightarrow Z Z^{*}$

The Higgs boson decay to four leptons, $H \rightarrow Z Z \rightarrow 4 l$, provides good sensitivity to the measure its properties despite the small branching ratio $(\sim 0.0125 \%)$ due to the low background contribution 

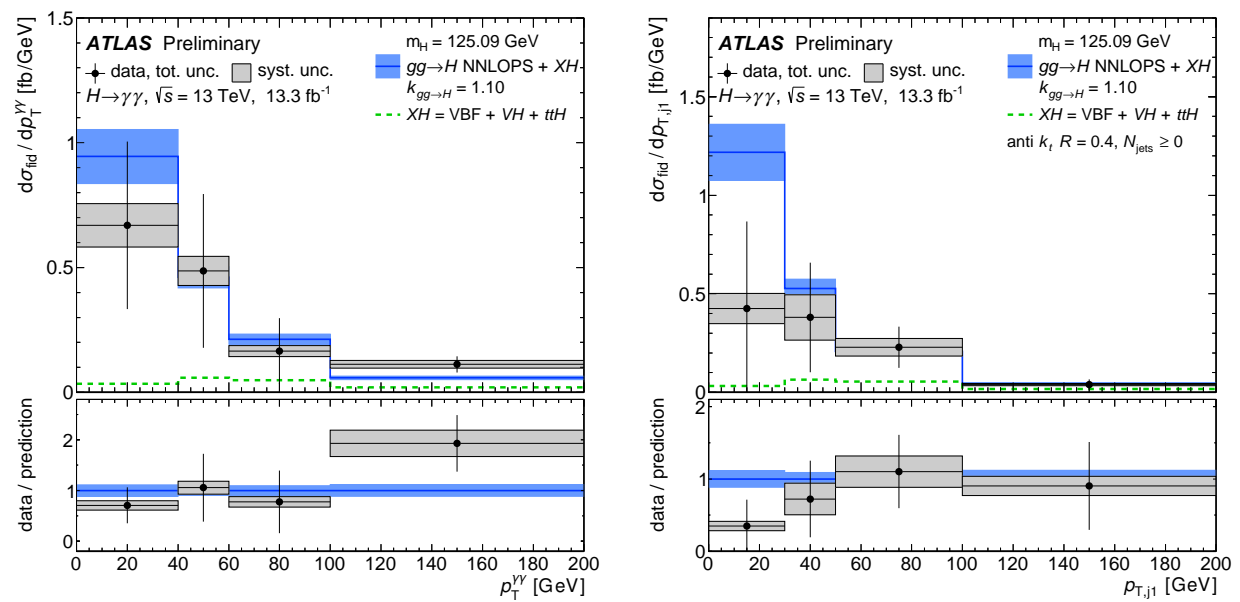

Figure 2: ATLAS differential cross section measurements for $p p \rightarrow H \rightarrow \gamma \gamma$ as a function of the diphoton transverse momentum (left) and as a function of the leading jet transverse momentum (right). More differential studies can be found at [8].

which mainly consists of $Z Z \rightarrow 4 l$. This analysis is optimized for different production modes as well. Figure 3 shows the $m_{4 l}$ distributions from ATLAS and CMS with $14.8 \mathrm{fb}^{-1}$ and $12.9 \mathrm{fb}^{-1}$ of pp collision data, respectively [12][11]. The measured fiducial cross sections are shown in Table 2. CMS obtains a relative precision on the fiducial measurement of about $30 \%$, similar to the final CMS Run 1 precision. Differential cross section measurements are done by CMS and some are shown in Fig. 4 [11].
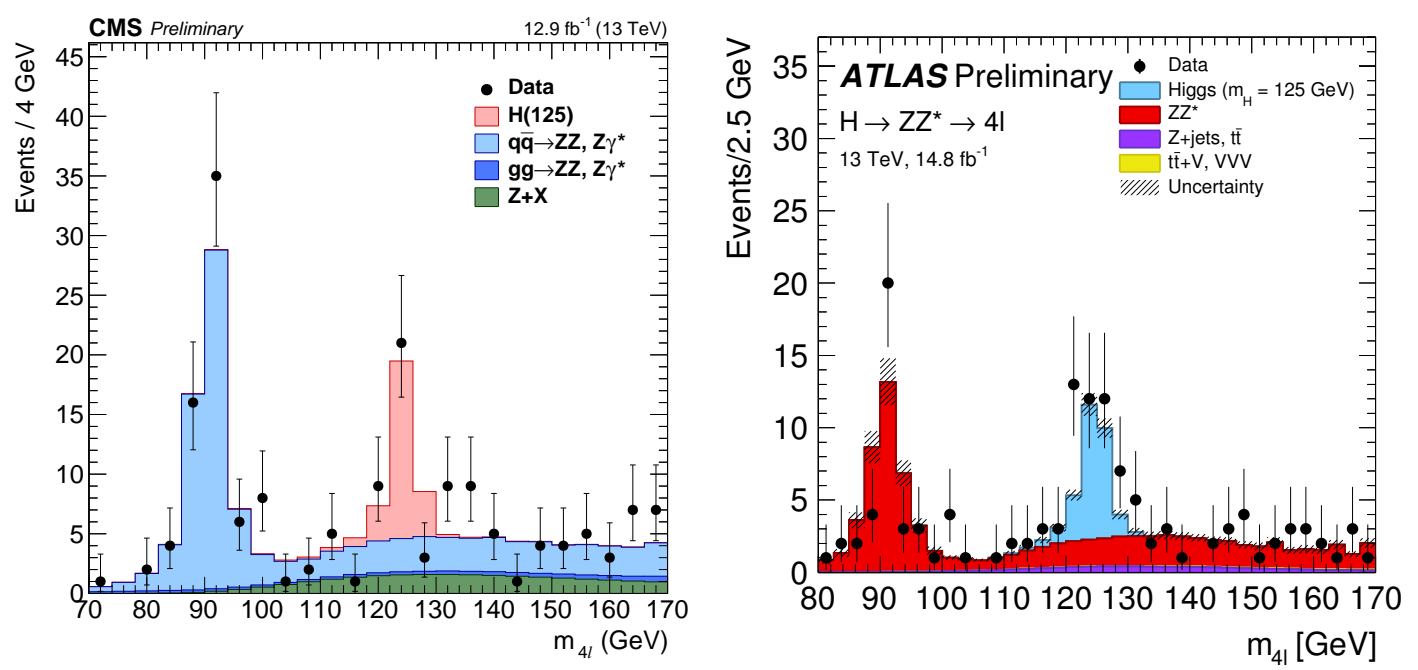

Figure 3: CMS (left) and ATLAS (right) four-lepton reconstructed invariant mass, $\mathrm{m}_{4 l}$, compared to the background expectation. [11][12]. 
Table 2: Fiducial cross section measurements $H \rightarrow Z Z^{*}[11,12]$

$\begin{array}{lll}\sqrt{s}=13 \mathrm{TeV} & \text { Measurement }(\mathrm{fb}) & \text { SM prediction }(\mathrm{fb}) \\ \text { ATLAS }\left(14.8 \mathrm{fb}^{-1}\right) & 4.54_{-0.90}^{+1.02} & 3.07_{-0.25}^{+0.21} \\ \text { CMS }\left(12.9 \mathrm{fb}^{-1}\right) & 2.29_{-0.64}^{+0.74}(\mathrm{stat})_{-0.23}^{+0.30}(\mathrm{syst}) & 2.53 \pm 0.13\end{array}$
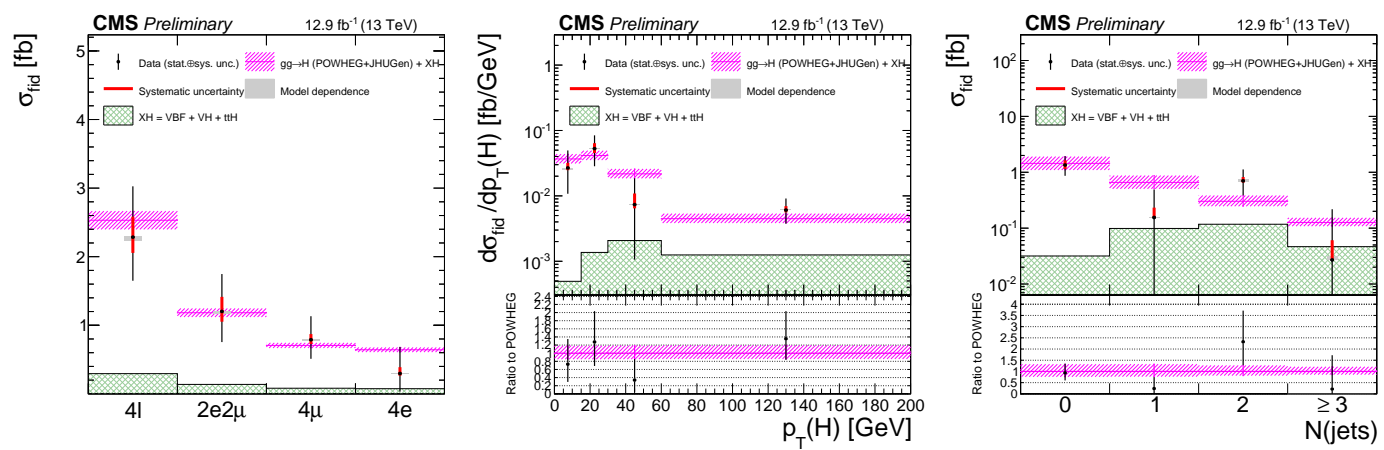

Figure 4: (left) CMS measurement of the fiducial cross sections in each final state. CMS result of the differential cross section measurement for $p_{T}(\mathrm{H})$ (middle) and $\mathrm{N}(\mathrm{jets})$ (right). The acceptance and theoretical uncertainties in the differential bins are are calculated using POWHEG. [11]

\subsection{Combination of $H \rightarrow \gamma \gamma$ and $H \rightarrow Z Z^{*}$}

ATLAS combines the measurements in the $\gamma \gamma$ and $4 l$ channels at $m_{H}=125.09 \pm 0.24 \mathrm{GeV}$. Higgs boson production is observed with $10 \sigma$ significance (8.6 $\sigma$ expected) in agreement with SM expectations. The fiducial cross section is measured to be $59.0_{-9.2}^{+9.7}(\mathrm{stat})_{-3.5}^{+4.4}$ (syst) pb while the SM predicts $55.5_{-3.4}^{+2.4} \mathrm{pb}$. The signal strength is measured to be $\mu=1.13_{-0.17}^{+0.18}$ [13] which is comparable with the achieved precision from Run 1. Figure 5 shows the contours in the $(\sigma B) / B_{S M}$ plane for $g g F$ and $V B F$ production modes as measured in $H \rightarrow \gamma \gamma$ and $H \rightarrow Z Z^{*}$, together with the SM prediction using ATLAS data. Also shown in Fig. 5 are the measured cross sections for these channels as a function of $\sqrt{s}$.
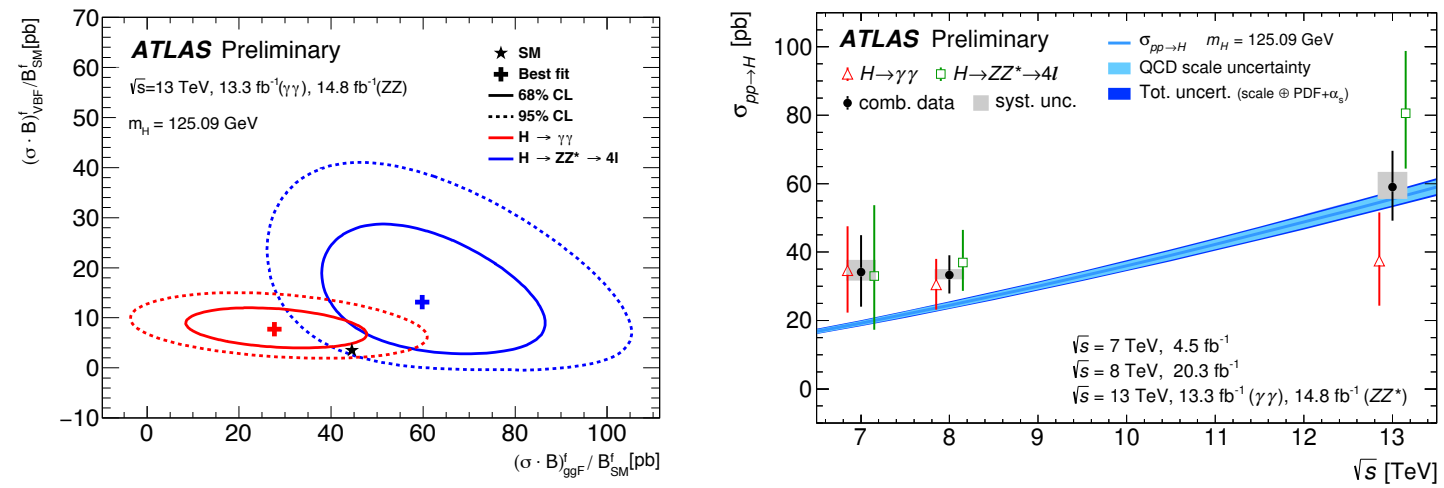

Figure 5: (left) Contours in the $(\sigma B)_{g g F}^{f} / B_{S M}^{f}-(\sigma B)_{V B F}^{f} / B_{S M}^{f}$ plane as measured in $H \rightarrow \gamma \gamma$ and $H \rightarrow Z Z^{*}$, together with the SM prediction. (right) Total $p p \rightarrow H+X$ cross sections measured at different center-ofmass energies compared to SM predictions at up to N3LO in QCD [13]. 


\section{$2.4 t \bar{t} H$}

In the SM, the Higgs boson is expected to have a particularly large Yukawa coupling to the top quark. Indirectly, this coupling is accessible via fermion loops which occur in the gluon fusion production process and the Higgs decay to photon pairs. For an unambiguous measurement, it is important to probe the top-Higgs coupling directly. This is possible through the measurement of Higgs production in association with top quark pairs. The signal is extracted according to the decay of the Higgs boson to $W W, Z Z, \tau \tau, b \bar{b}$ and $\gamma \gamma$. The latter one is included in the analysis of $H \rightarrow \gamma \gamma$ above mentioned. The other decays are grouped in $t \bar{t} H(H \rightarrow b b)$ and $t \bar{t} H(H \rightarrow$ multileptons $)$ decays.

\subsection{1 $t \bar{t} H(H \rightarrow b \bar{b})$}

Among the $t \bar{t} H$ channels, the ones with the Higgs boson decays into a $b \bar{b}$ pair have the largest branching ratio, offering also sensitivity to the Higgs-Bottom Yukawa coupling. The measurement of $t \bar{t} H(H \rightarrow b \bar{b})$ in the single-lepton and dilepton channels is updated by ATLAS using $13.2 \mathrm{fb}^{-1}$ pp collision data. To maximise the signal sensitivity the events are categorised according to their number of jets and $b$-jets. Figure 6 shows predictions of the event yields after the fit to the data. The measured signal strength obtained in the dilepton channel is $\mu=4.6_{-2.3}^{+2.9}$ and single lepton is $\mu=1.6 \pm 1.1$ which combined result in $\mu=2.1_{-0.9}^{+1.0}$ [14]. Results using 2015 dataset from the CMS experiment can be found in Ref. [15]. Currently the dominant systematic uncertainty in both experiments is the signal and background modeling and normalization which is found to be larger than statistical uncertainty.
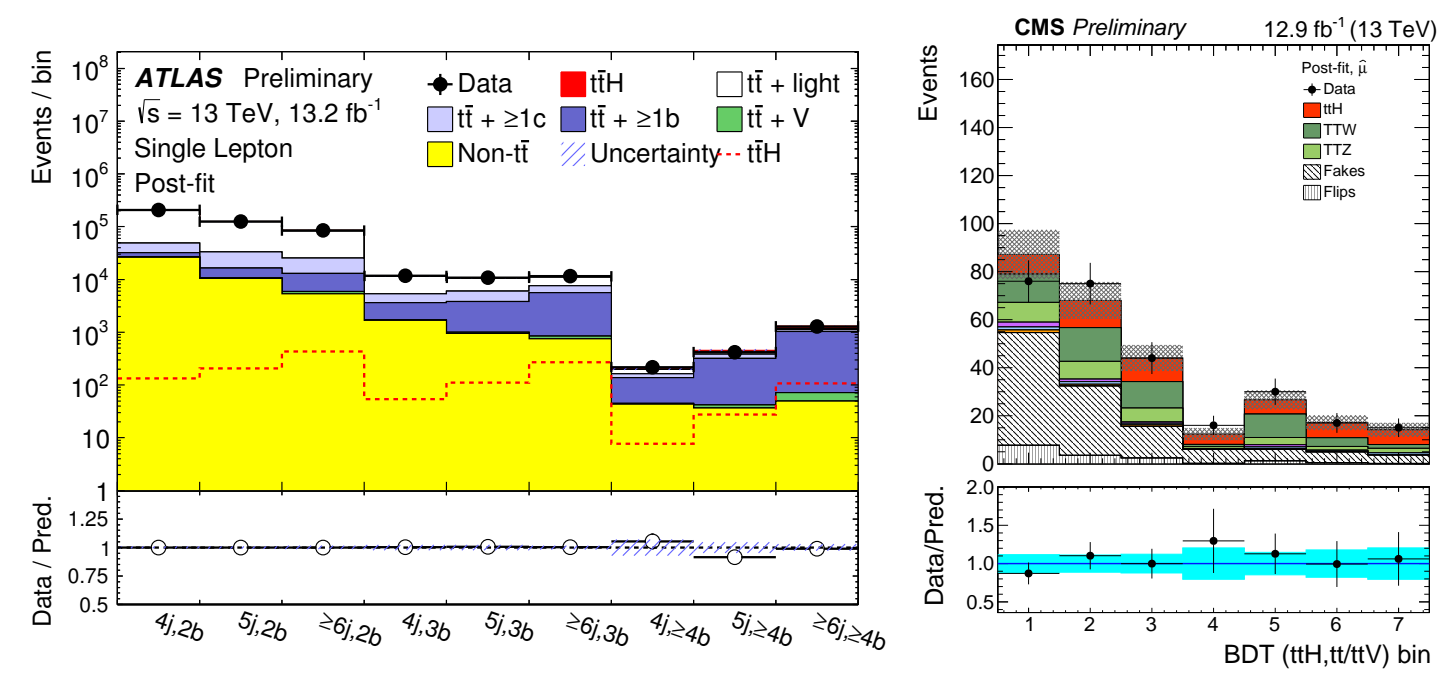

Figure 6: (left) ATLAS results on $t \bar{t} H(H \rightarrow b \bar{b})$. Comparison of predicted and observed event yields in the single-lepton channel after the fit to the data [14]. (right) Combination of the BDT classifier outputs in the bins used for signal extraction for the same-sign dilepton channel in the CMS analysis of $t \bar{t} H(H \rightarrow$ multileptons). Post-fit distributions and uncertainties are shown. [17]

\subsection{2 $t \bar{t} H(H \rightarrow$ multileptons $)$}

Both experiments, ATLAS and CMS, update results on $t \bar{t} H$ with $H$ decaying to leptons via $W W, Z Z$, and $\tau \tau$, with $13.2 \mathrm{fb}^{-1}$ and $12.9 \mathrm{fb}^{-1}$ of collected data, respectively. These analyses 
focus mainly on final states with clean signatures and low backgrounds. The signal region includes two or more leptons, with at least one tagged $b$-jet, and allows for at least one hadronic $\tau$ decay. The dominant systematic uncertainties are the fake-rate measurements and non-prompt background estimates. Figure 6 shows a BDT classifier output in the bins used for signal extraction for the CMS same-sign dilepton channel. The measured signal strength obtained by ATLAS is $\mu=2.5_{-1.1}^{+1.3}$ and by CMS is $\mu=2.0_{-0.9}^{+0.8}[16,17]$. ATLAS combined results from the $H \rightarrow b \bar{b}$ and $H \rightarrow$ multileptons channels obtaining a signal strength of $\mu=1.7_{-0.8}^{+0.7}[18]$

\section{$2.5 t H(H \rightarrow b b)$}

The smallest SM production cross section of Higgs bosons is associated production with single-top quarks. In the SM interfering diagrams make this cross section smaller than could be reached in Run 2. Nevertheless, under an inverted top-coupling (ITC) scenario the cross section would be largely enhanced. CMS has established upper limits using the 2015 dataset that are 113.7 x SM (98.6 expected). Under the ITC assumption, the observed upper limit x SM is found to be 6.0 (6.4 expected) [19].

\section{6 $V H(H \rightarrow b \bar{b})$}

The decay $H \rightarrow b \bar{b}$ has the largest branching ratio $\left(57.7 \%\right.$ at $\left.m_{H}=125 \mathrm{GeV}\right)$ in the SM, but suffers from a large QCD background. The main search for Higgs-bottom coupling is production associated with a vector boson $(V H)$ with $V=W, Z$. This translates in an improved signature with easier triggering via leptons and missing transverse energy and also an improved signal-tobackground ratio. ATLAS updates a search in this channel with $13.2 \mathrm{fb}^{-1}$ of pp collision data using a multivariate technique to extract the signal with most of the discrimination arising from the $m_{b \bar{b}}$ and $\Delta R(b 1, b 2)$ variables. The combined measured relative signal strength for the $V H$ channel is found to be $\mu=0.21_{-0.50}^{+0.51}$, in good agreement with the SM. This corresponds to an observed $0.4 \sigma$ ( $1.94 \sigma$ expected). The fit is tested by measuring the $W Z$ cross section with $Z \rightarrow b \bar{b}$ which is measured to be $\mu=0.91 \pm 0.17$ (stat) ${ }_{-0.27}^{+0.32}$ (syst) with a corresponding observed significance of $3.0 \sigma$ (expected $3.2 \sigma)[20]$.

\section{$2.7 \operatorname{VBF}(H \rightarrow b \bar{b})$}

The $H \rightarrow b \bar{b}$ production strength is also analyzed in vector boson fusion $(V B F)$ process. In $V B F$, quarks from the initial protons emit $W$ or $Z$ bosons, which then fuse to form the Higgs boson. The final state is characterized by the decay products of the Higgs boson and two hadronic jets with a large invariant mass and a large difference in rapidity. CMS presents a new result using 2015 dataset of $2.3 \mathrm{fb}^{-1}$ [21]. A signal significance of $2.2 \sigma$ is observed, while $0.8 \sigma$ is expected. Additionally, ATLAS sets limits on this production mode by also requiring a high $p_{T}$ photon, which provides a clean signature for efficient triggering, of $4 \times \mathrm{SM}$ (expected $6 \times \mathrm{SM}$ ) with $12.6 \mathrm{fb}^{-1}$ of collected data [22]. Figure 7 shows the spectrum of the invariant mass of the two $b$-jets from a specific category of events for the CMS and ATLAS results.

\section{$2.8 H \rightarrow \mu \mu$}

The branching ratio of $H \rightarrow \mu \mu$ is very small and the signal is overwhelmed by the Drell-Yan $Z \rightarrow \mu \mu$ background. ATLAS updates a search for this decay using $13.2 \mathrm{fb}^{-1}$ of pp collision data. 

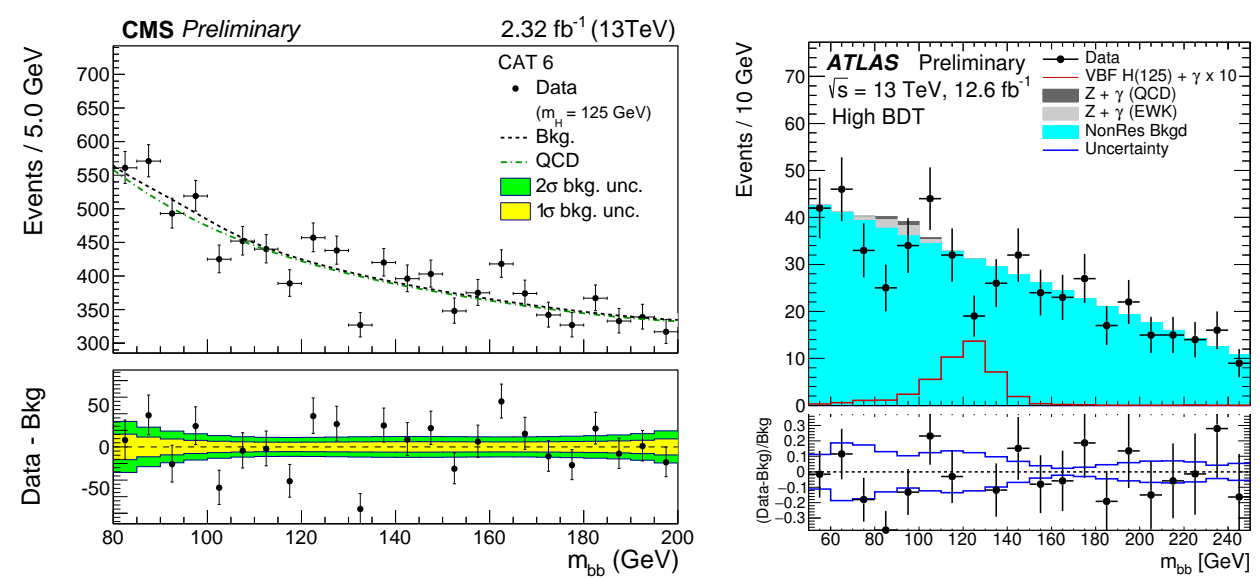

Figure 7: Fit of the invariant mass of the two b-jet candidates for the Higgs boson signal. (left) In the three event categories of double b-tagged in the background-only hypothesis for the CMS analysis [21]. (right) Events in the highest BDT region considered in the likelihood fit in the ATLAS analysis [22].

The analysis focuses on the $V B F$ production and is divided into several bins of Higgs boson $p_{T}$. The observed upper limit (at 95\% CL) of the signal strength is ssset to 4.4*SM (5.5 expected) [23].

\section{BSM Higgs boson searches}

A multitude of BSM Higgs searches are carried out at CMS and ATLAS at $\sqrt{s}=13 \mathrm{TeV}$. The highlights of a few search results are given below.

\subsection{Heavy Higgs boson to vector bosons: $H \rightarrow Z Z, H \rightarrow W W$, and $H \rightarrow \tau \tau$}

ATLAS and CMS present searches for the production of a heavy neutral scalar Higgs boson decaying into two SM $Z$ bosons with $14.8 \mathrm{fb}^{-1}$ and $12.9 \mathrm{fb}^{-1}$ of pp collision data, respectively. The results of these searches are interpreted in the scenario of a heavy Higgs boson with a width that is small compared with the experimental mass resolution and assuming that it is produced via the $g g F$ and $V B F$ processes. The invariant mass of the four leptons in the $Z Z \rightarrow 4 l$ channels for both experiments is shown in Fig. 8. No signal is observed and upper limits at 95\% CL are set for different decay-width assumptions [24, 25].

High-mass searches in $H \rightarrow W W$ with subsequent leptonic decays of the $W$ bosons are also pursued by ATLAS and CMS and can be found at [26] and [27]. Other searches in the diboson ZZ and $W W$ channels are included in the "Exotic searches at the LHC" talk in this conference.

In the Minimal Supersymmetric Standard Model (MSSM) a heavy Higgs boson can have enhanced couplings to down-type fermions causing an increased production of the heavy Higgs boson that decays mainly to $b$ quarks and $\tau$ leptons. A new ATLAS search for $H \rightarrow \tau \tau$ which includes new triggers and event categories uses $13.2 \mathrm{fb}^{-1}$ of collected data. Figure 8 shows the transverse invariant mass calculated with the momentum of the $\tau_{\mathrm{s}}$ and missing transverse energy. The data is in good agreement with the SM expectations. Upper limits at 95\% CL are set for MSSM and hMSSM benchmark scenarios and show an improved sensitivity with respect to previous limits [28]. 

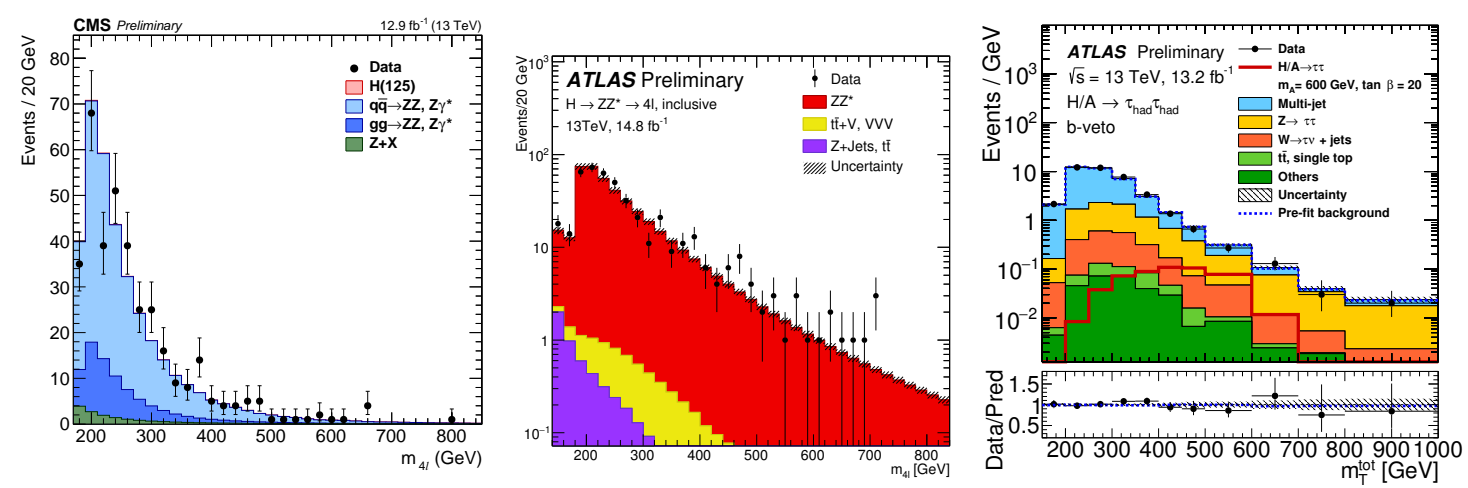

Figure 8: CMS (left) and ATLAS (middle) distribution of the four-lepton reconstructed invariant mass $m_{4 l}$ in the high-mass range [24, 25]. (right) The distribution of $m_{T}^{\text {tot }}$ for the $b$-veto channel in the $H / A \rightarrow \tau \tau$ search [28].

\subsection{Heavy Higgs to vector bosons: $H \rightarrow h h \rightarrow b b \tau \tau$}

In the SM, the production cross section for Higgs-boson pairs is extremely small. On the contrary, this pair production is enhanced in many BSM scenarios. Searches for a heavy neutral scalar Higgs $(\mathrm{H})$ decaying into a pair of SM Higgs (h) thus offer great potential in the search for new phenomena. New searches for a heavy neutral Higgs-bosons decaying to di-Higgs bosons are done at $\sqrt{s}=13 \mathrm{TeV}$. Data and predictions from the SM are in agreement within systematic uncertainties.

$H \rightarrow h h \rightarrow b b \tau \tau$ : CMS presents a search using $12.9 \mathrm{fb}^{-1}$ of collected data and selects the three most sensitive decay channels of the $\tau$ leptons, namely $e \tau_{h}, \mu \tau_{h}$, and $\tau_{h} \tau_{h}$, where $\tau_{h}$ indicates a $\tau$ lepton decay involving hadrons. At high $m_{H}$ the analysis uses substructure information for jets. Model-independent upper limits on the values of the cross section times branching ratio are derived for different signal mass hypotheses [29]. A non-resonant search is done in the same channel and limits as a function of the ratio of the anomalous trilinear coupling to the SM trilinear coupling $\left(\kappa_{\lambda}=\lambda_{\text {hhh }} / \lambda_{\text {SMhhh }}\right)$ are obtained [30].

$H \rightarrow h h \rightarrow b b W W$ : CMS reports a search using $2.3 \mathrm{fb}^{-1}$ of pp collision data selecting the leptonic decays of the $W$ bosons. The search focuses on the invariant mass distribution of the $b$-jet pair combined with a BDT discriminant based on kinematic infromation. Upper limits at 95\% CL on the resonant Higgs boson pair production cross section times branching fraction are computed as a function of $m_{H}$ for the spin- 0 and spin-2 hypotheses [33].

$H \rightarrow h h \rightarrow b b b b:$ ATLAS and CMS present searches that exploit the dominant $h \rightarrow b \bar{b}$ decay mode, with the four $b$-jets either reconstructed as distinct jets, or as a pair of large-radius jets. The sensitivity of these analysis is increased by including events with boosted topologies. ATLAS (CMS) results are carried out with 13.3. $\mathrm{fb}^{-1}\left(2.3 \mathrm{fb}^{-1}\right)$ of LHC pp collision data. No significant excess of events is observed above the estimated background, and limits are set on the cross section times branching ratio as a function of $m_{H}$ for resonant production [31, 32]. ATLAS also constrains the non-resonant SM Higgs-boson pair production to be less than $330 \mathrm{fb}, 29$ times the SM predicted cross section of $11.3_{-1.0}^{+0.9} \mathrm{fb}$. 


\subsection{Charged Higgs boson}

Charged Higgs bosons appear in many extensions of the SM with at least two Higgs doublets. Many searches are done by ATLAS and CMS with the Run 2 data collected.

$H^{ \pm} \rightarrow W Z$ : CMS searches for this process in a data sample of integrated luminosity corresponding to $15.2 \mathrm{fb}^{-1}$. Events are selected with 3 leptons, two jets with large rapidity separation and high dijet mass, and moderate missing transverse energy. Results are interpreted in the context of Higgs triplet models [34].

$H^{ \pm} \rightarrow t b$ : ATLAS searches for charged Higgs bosons heavier than the top quark in $13.2 \mathrm{fb}^{-1}$ of pp collision data using multi-jet final states with one electron or muon from the top-quark decay. Results are interpreted in benchmark scenarios of the MSSM. [35].

$H^{ \pm} \rightarrow \tau+$ jets: ATLAS searches for charged Higgs bosons in $14.7 \mathrm{fb}^{-1}$ of data targeting the $\tau+$ jets channel in top-quark-associated $H^{ \pm}$production with a hadronically decaying $W$ boson and $\tau$ lepton in the final state. Results are interpreted in the hMSSM benchmark scenario [36].

In all these searches, the observation agrees with the SM prediction. Limits on the charged Higgs boson cross section times branching fraction are given as a function of the charged Higgs boson mass.

\subsection{Invisible Higgs boson decays}

Models like SUSY predict dark matter particles that may couple to the Higgs boson. Direct searches for the $H \rightarrow$ invisible decay are carried out by CMS in the $g g F, V B F$ and $V H$ production modes. The search includes events in which a leptonically decaying $Z$ boson and hadronically decaying $W$ or $Z$ are boson produced in association with large missing transverse momentum [37, $38,39]$. These searches are based on a 2016 data sample corresponding to an integrated luminosity of $12.9 \mathrm{fb}^{-1}$. Results are combined assuming SM values for ratios of production cross-sections and yield an observed $\mathrm{BR}(H \rightarrow$ invisible $)<24 \%(0.23 \%$ expected $)$.

\section{Summary}

The large sample of events available in Run 2 of the LHC at $\sqrt{s}=13 \mathrm{TeV}$ has allowed ATLAS and CMS to establish the Higgs boson production and decay modes with similar sensitivity as previously acchieved with Run 1 data. The extensive studies of the Higgs-boson properties which have been performed using Run 1 data will soon start to be surpassed by results from Run 2 . The current increase in data is opening up new channels to study the interactions of Higgs bosons and SM particles. Moreover, the higher collision energies extend the reach of ATLAS and CMS experiments to direct probe for BSM physics by searching for additional Higgs bosons and for anomalous decays of the Higgs boson.

\section{References}

[1] ATLAS Collaboration, Phys. Lett. B 716, 1, 2012.

[2] CMS Collaboration, Phys. Lett. B 716, 30, 2012.

[3] ATLAS and CMS Collaborations, ATLAS-CONF-2015-044/CMS-PAS-HIG-15-002 (2015). 
[4] ATLAS and CMS Collaborations, Phys. Rev. Lett. 114, 191803 (2015).

[5] ATLAS Collaboration, Eur. Phys. J. C75 (2015) 476.

[6] CMS Collaboration, Phys. Rev. D 92 (2015) 012004.

[7] CMS Collaboration, CMS-PAS-HIG-16-020 (2016).

[8] ATLAS Collaboration, ATLAS-CONF-2016-067 (2016).

[9] CMS Collaboration, EPJC74 no. 10 (2014).

[10] ATLAS Collaboration, Phys. Rev. D90 112015 (2014).

[11] CMS Collaboration, CMS-PAS-HIG-16-033 (2016).

[12] ATLAS Collaboration, ATLAS-CONF-2016-079 (2016).

[13] ATLAS Collaboration, ATLAS-CONF-2016-081 (2016).

[14] ATLAS Collaboration, ATLAS-CONF-2016-080 (2016).

[15] CMS Collaboration, CMS-PAS-HIG-16-004 (2016).

[16] ATLAS Collaboration, ATLAS-CONF-2016-058 (2016).

[17] CMS Collaboration, CMS-PAS-HIG-16-022 (2016).

[18] ATLAS Collaboration, ATLAS-CONF-2016-068 (2016).

[19] CMS Collaboration, CMS-PAS-HIG-16-019 (2016).

[20] ATLAS Collaboration, ATLAS-CONF-2016-091 (2016).

[21] CMS Collaboration, CMS-PAS-HIG-16-003 (2016).

[22] ATLAS Collaboration, ATLAS-CONF-2016-063 (2016).

[23] ATLAS Collaboration, ATLAS-CONF-2016-041 (2016).

[24] CMS Collaboration, CMS-PAS-HIG-16-033 (2016).

[25] ATLAS Collaboration, ATLAS-CONF-2016-079 (2016).

[26] CMS Collaboration, CMS-PAS-HIG-16-023 (2016).

[27] ATLAS Collaboration, ATLAS-CONF-2016-074 (2016).

[28] ATLAS Collaboration, ATLAS-CONF-2016-085 (2016).

[29] CMS Collaboration, CMS-PAS-HIG-16-029 (2016).

[30] CMS Collaboration, CMS-PAS-HIG-16-028 (2016).

[31] ATLAS Collaboration, ATLAS-CONF-2016-049 (2016).

[32] CMS Collaboration, CMS-PAS-HIG-16-002 (2016).

[33] CMS Collaboration, CMS-PAS-HIG-16-011 (2016).

[34] CMS Collaboration, CMS-PAS-HIG-16-027 (2016).

[35] ATLAS Collaboration, ATLAS-CONF-2016-089 (2016).

[36] ATLAS Collaboration, ATLAS-CONF-2016-088 (2016).

[37] CMS Collaboration, CMS-PAS-HIG-16-016 (2016).

[38] CMS Collaboration, CMS-PAS-EXO-16-037 (2016).

[39] CMS Collaboration, CMS-PAS-EXO-16-038 (2016). 